\title{
Plasmonic Bowtie Nanolaser Arrays
}

\author{
Jae Yong Suh, ${ }^{\dagger}$ Chul Hoon Kim, ${ }^{\dagger, \S, \|}$ Wei Zhou, ${ }^{\ddagger}$ Mark D. Huntington, ${ }^{\ddagger}$ Dick T. Co, ${ }^{\dagger, \S}$ \\ Michael R. Wasielewski, ${ }^{\dagger, \S}$ and Teri W. Odom ${ }^{*} \dagger, \ddagger, \S$
}

${ }^{\dagger}$ Department of Chemistry, Northwestern University, Evanston, Illinois 60208, United States

${ }^{\ddagger}$ Department of Materials Science and Engineering, Northwestern University, Evanston, Illinois 60208, United States

${ }^{\S}$ Argonne-Northwestern Solar Energy Research (ANSER) Center, Northwestern University, Evanston, Illinois 60208, United States

"Max Planck Center for Attosecond Science, POSTECH, Pohang, South Korea 790-784

\section{Supporting Information}

ABSTRACT: Plasmonic lasers exploit strong electromagnetic field confinement at dimensions well below the diffraction limit. However, lasing from an electromagnetic hot spot supported by discrete, coupled metal nanoparticles (NPs) has not been explicitly demonstrated to date. We present a new design for a room-temperature nanolaser based on threedimensional (3D) Au bowtie NPs supported by an organic gain material. The extreme field compression, and thus ultrasmall mode volume, within the bowtie gaps produced laser oscillations at the localized plasmon resonance gap mode of the 3D bowties. Transient absorption measurements confirmed ultrafast resonant energy transfer between photoexcited dye molecules and gap plasmons on the picosecond time scale. These plasmonic nanolasers are anticipated to be readily integrated into Si-based photonic devices, all-optical circuits, and nanoscale biosensors.

KEYWORDS: Localized surface plasmons, plasmon lasing, coupled metal nanoparticles, stimulated emission, Purcell effect
$\mathrm{T}$ he realization of coherent photon sources on the nanometer scale is a major challenge and a key requirement for ultrafast optical applications. Miniaturizing photonic cavities is difficult because the diffraction limit requires the size of the optical resonator $(L)$ to be greater than half the lasing wavelength $\left(L>\left(\lambda / 2 n_{\text {eff }}\right)\right)$, where $n_{\text {eff }}$ is the effective refractive index of the laser medium. ${ }^{1}$ One route to achieve lasing from subwavelength resonators is to exploit localized surface plasmons (LSPs) that can be excited in metal NPs. ${ }^{2,3}$ When a gain material overlaps spatially and spectrally with the LSP fields, lasing can occur when the gain is high enough to compensate metal losses at the plasmon frequency. ${ }^{4,5}$ Although nanoscale lasing has been observed from Au colloids surrounded by dye-doped dielectric shells, ${ }^{6}$ disadvantages include the exponential decay of optical fields from single NPs and lack of ultrafast kinetic information from time-resolved emission. Plasmonic lasing has also been reported in a variety of nanostructured inorganic semiconductors involving flat metal surfaces. ${ }^{7-12}$ In these architectures, however, either cryogenic temperatures or high pump powers were required to overcome large Joule losses from the planar metal films, thereby preventing facile application of the nanolasers.

Bowtie resonators-metal NP dimers with a gap size of tens of nanometers-are an ideal system to localize and enhance electric fields within nanoscale volumes. The primary advantage of a bowtie geometry over a symmetric, single NP as an optical resonator is their high Purcell factor $F=\left(3 / 4 \pi^{2}\right)\left(Q / V_{\text {eff }}\right)(\lambda /$ $\left.2 n_{\text {eff }}\right)^{3}$, where $Q$ is the quality factor and $V_{\text {eff }}$ is the effective mode volume. Finite-difference time-domain (FDTD) simulations have shown that metal bowties support high field enhancements $\left(|E|^{2} /\left|E_{0}\right|^{2} \equiv 10^{3}\right.$ to $\left.10^{4}\right)$ in a nanometer-sized gap, ${ }^{13-16}$ which result in a reduction in the mode volume. ${ }^{17,18}$ With an increased Purcell factor because of a reduced mode volume, the threshold condition to initiate lasing in bowtie NPs can be readily satisfied, even at ambient temperatures. ${ }^{4,19}$ Here we show that plasmonic lasing can occur at room temperature near LSP resonance wavelengths when strong electric field localizations (hot spots) are present in the gap.

Figure 1a depicts an array of 3D Au bowtie NPs that exhibits SP-assisted lasing. Each unit bowtie supports a strong LSP resonance within the gap. Photoexcited molecules in the gain medium can couple to the bonding (gap) LSP mode when the photoluminescence (PL) spectrum of the dye overlaps with the LSP band. Resonant energy transfer (RET) from the excited state (ES) of the dye to the gap plasmons can support laser oscillations when a certain pump threshold is achieved (Figure 1b). Since the bowties are arranged in a two-dimensional (2D) periodic array, surface-emission lasing (blue arrow) at the band-

Received: August 19, 2012

Revised: September 26, 2012

Published: September 26, 2012 


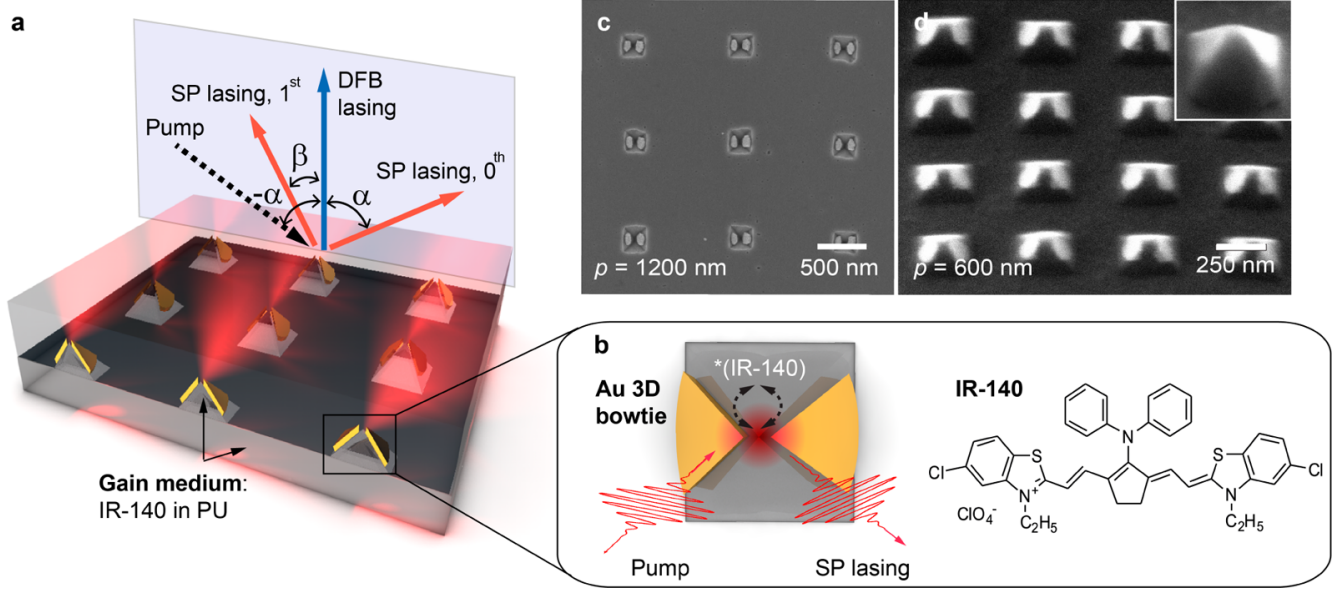

Figure 1. Scheme of 3D bowtie plasmonic lasers. (a) Nanolaser structure consists of a gain medium slab (IR-140 dye in polyurethane (PU), $n \sim$ 1.52) supporting a semi-infinite array of Au bowties and dielectric overlayer $(n=1.52)$. Output direction of surface plasmon (SP) lasing follows a phase grating equation where $-\alpha$ is the angle of pump beam and $\alpha$ and $\beta$ are the output angles of 0th and 1st diffraction-order SP lasing. (b) Pumping at $800 \mathrm{~nm}$ creates photoexcited IR-140 dye molecules in PU, and lasing occurs as a result of ES-LSP coupling. (c) Top-down SEM image shows Au bowties in Si pits with $p=1200 \mathrm{~nm}$. (d) Au bowties on IR-140/PU substrate with $p=600 \mathrm{~nm}$. Inset shows a perspective image of a unit bowtie.
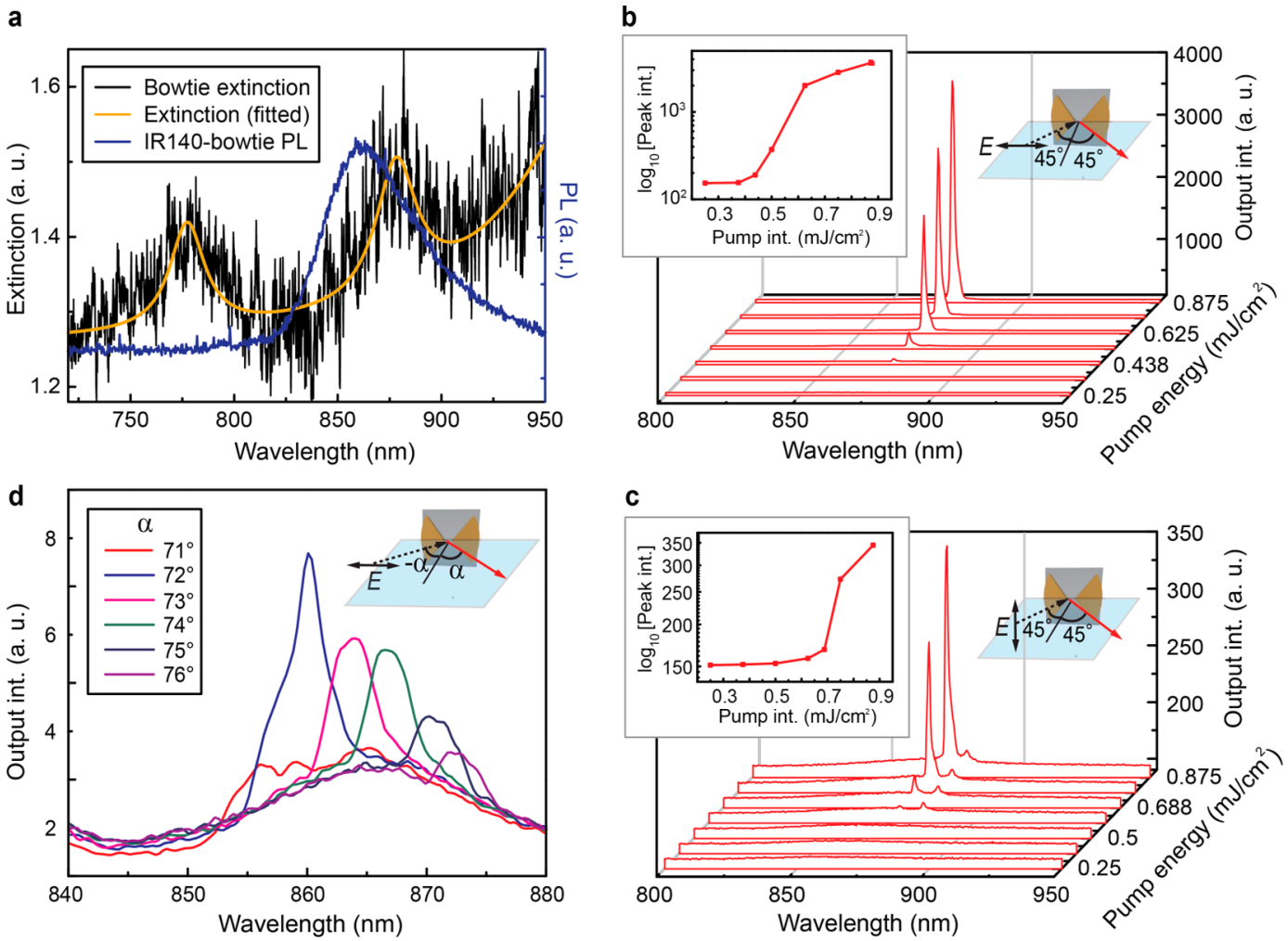

Figure 2. Spectral overlap between gain and gap LSP resonance affects strength of SP laser oscillation. (a) PL spectrum of IR-140 and extinction spectrum of Au bowties with $p=1200 \mathrm{~nm}$. The PL line width is $\Gamma_{\mathrm{PL}}$, and the bonding LSP mode with width $\Delta \omega_{\mathrm{SP}}$ spectrally overlaps with the PL band. Evolution of lasing spectra from 3D Au bowties under pump polarization (b) parallel and (c) perpendicular to the dimer axis. (insets) Peak emission intensity vs pump pulse energy density plots on a semilogarithmic scale show threshold levels, where the slopes change from spontaneous emission to lasing to gain-saturation. (d) Amplification of lattice plasmons can occur when SP out-coupling is allowed according to the energy dispersion.

edge can also be present. Figure 1c shows a top-down scanning electron microscopy (SEM) image of 3D Au bowties in a Si pyramidal template with $p=1200 \mathrm{~nm}$ and gap distance $(d)=$ $35 \mathrm{~nm}$. The gap distance was controlled such that the frequency of the bonding LSP mode ${ }^{14}$ was resonant with the $S_{1} \rightarrow S_{0}$ emission of the selected dye molecule. Fabrication details of 3D
$\mathrm{Au}$ bowties as well as their linear and nonlinear optical properties were reported in ref 14 . Figure $1 \mathrm{~d}$ presents an array of Au bowtie resonators with $p=600 \mathrm{~nm}$ and $d=25 \mathrm{~nm}$ supported on polyurethane (PU) doped with the IR-140 dye; the volume density $(N)$ of dye molecules was measured to be $4.4 \times 10^{18} \mathrm{~cm}^{-3}$ by UV-vis optical absorption measurements. 

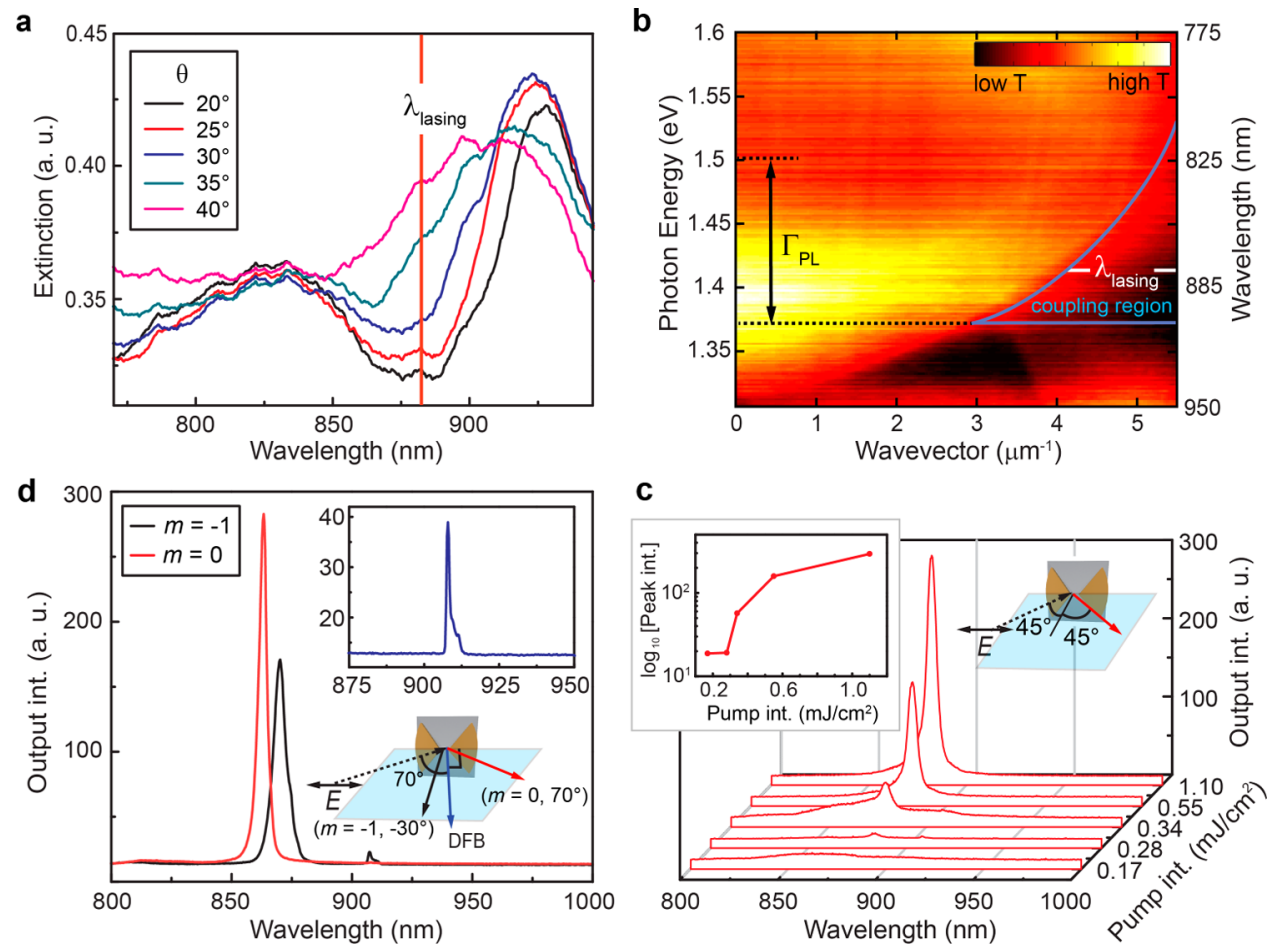

Figure 3. ES-plasmon coupling region defined by gap and lattice properties of bowties. (a) Extinction spectra of $p=600 \mathrm{~nm}$ Au bowties on a IR140/PU slab. The peak at $830 \mathrm{~nm}$ is the IR-140 absorption band, and only the antibonding mode that emerges above $\theta>35^{\circ}$ overlaps with the gain. (b) Energy dispersion diagram, where the RA PU $(0, \pm 1)$ mode (upper blue curve) cuts off the LSP band, showing the ES-plasmon coupling region defined by the spectral overlap with IR-140 PL band. (c) $p=600 \mathrm{~nm}$ bowties exhibit an order of magnitude smaller output intensity and four times wider fwhm of the emission spectra compared to $p=1200 \mathrm{~nm}$. (inset) Peak emission intensity vs pump pulse energy density. (d) For $p=600 \mathrm{~nm}$, two diffraction modes $(m=0$ and $m=-1)$ are found at $70^{\circ}$ and $-30^{\circ}$, respectively, with $\alpha=-70^{\circ}$. (inset) Small DFB emission is detected at 910 $\mathrm{nm}$, which satisfies the second-order DFB lasing condition. The $p=1200$ array in Figure 2 does not support DFB lasing because the DFB lasing wavelength is located outside the PL band of IR-140.

Using the emission cross-section $\left(\sigma_{\mathrm{e}}\right)$ in ref 20, the optical gain $\left(N \sigma_{\mathrm{e}}\right)$ in the absence of metal nanostructures is estimated to be $2640 \mathrm{~cm}^{-1}$. The cavity-enhanced stimulated emission crosssection $\left(\sigma_{\mathrm{e}}^{\mathrm{c}}\right)$ when lasing is achieved is $F \cdot \sigma_{\mathrm{e}}{ }^{21}$

Figure $2 \mathrm{a}$ shows that the bonding gap plasmon resonance centered at $880 \mathrm{~nm}$ from $p=1200 \mathrm{~nm}$ bowties spectrally overlaps with the PL band of IR-140 centered at $855 \mathrm{~nm}$. The high-energy peak at $780 \mathrm{~nm}$ corresponds to the antibonding LSP mode that arises from repulsive charge interactions across the gap. ${ }^{22}$ The energy difference between the gain transition $\left(E_{\mathrm{PL}}\right)$ and gap plasmon resonance $\left(\hbar \omega_{\mathrm{SP}}\right)$ was measured as $E_{\mathrm{PL}}$ $-\hbar \omega_{\mathrm{SP}}=25 \mathrm{meV}$. We define the ES-plasmon coupling region as that where the energy difference $E_{\mathrm{PL}}-\hbar \omega_{\mathrm{SP}}$ falls within the sum of half-width-half-maximum (hwhm) of the two modes, 1/ $2\left(\Gamma_{\mathrm{PL}}+\hbar \Delta \omega_{\mathrm{SP}}\right)$. In this coupling region, the radiative emission rate increases because of the increased Purcell effect, ${ }^{13,23}$ and the lasing (walk-off) frequency is located between the gain transition and the LSP frequency maximum. ${ }^{4}$ The laser spectra from the Au bowtie array showed a clear onset of stimulated emission when the pump polarization was parallel to the dimer axis (Figure $2 \mathrm{~b}$ ). The samples were pumped by fs-laser pulses with a $4 \times 4 \mathrm{~mm}$ beam spot size. As the pump intensity increased, a sharp peak $(\mathrm{fwhm}=1.5 \mathrm{~nm})$ appeared at $873 \mathrm{~nm}$, and the output intensity was increased by 2 orders of magnitude over the underlying spontaneous emission. The slope change in the pump-emission data indicates that the transition from fluorescence to lasing occurred at a threshold pump pulse fluence of $0.4 \mathrm{~mJ} \mathrm{~cm} \mathrm{~cm}^{-2}$ (Figure $2 \mathrm{~b}$, inset). At pump

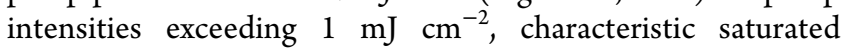

emission and dye bleaching were observed. We measured a different set of 3D bowtie resonators with $p=1200 \mathrm{~nm}$ and a smaller pump spot size of $1 \times 1 \mathrm{~mm}$ (Supporting Information, Figure S1). Lasing spectra equivalent to that in Figure $2 \mathrm{~b}$ were observed, but the output intensity was reduced because the number of bowties excited by the pump beam was lower.

When the pump beam was perpendicular to the dimer axis (Figure 2c), lasing at $873 \mathrm{~nm}$ was still observed, but the emission intensity was an order of magnitude smaller than that for parallel polarization. In addition to the narrow lasing at 873 $\mathrm{nm}$ associated with the bonding LSP mode, we also observed out-coupling of amplified lattice plasmons into emission angles $\alpha$ from $71^{\circ}$ to $76^{\circ}$ (Figure 2d). At high in-plane wavevectors $\left(k_{/ /}\right)$, lattice plasmons characterized as optical Bloch eigenmodes can propagate along the Rayleigh anomaly (RA) line in the energy dispersion. ${ }^{24,25}$ When the lattice plasmon mode hybridizes or spectrally overlaps with emitters in the gain, the propagating SP mode can be amplified and scattered as freespace photons at angles corresponding to $k_{/ / .}{ }^{26}$

To investigate the effects of overlap between the LSP frequency and the PL band on the SP-assisted lasing, we fabricated Au bowtie arrays with $d=25 \mathrm{~nm}$ and $p=600 \mathrm{~nm}$. Because of the smaller gap distance compared to bowties in Figure 2, the bonding LSP mode was red-shifted to $980 \mathrm{~nm}$ at normal incidence and could only be tuned to ca. $940 \mathrm{~nm}$ even at large $\theta=30^{\circ}$ (Figure 3a). In addition, the high-energy side of the bonding LSP band from 1.32 to $1.5 \mathrm{eV}$ (Figure $3 \mathrm{~b}$, dark region) was largely suppressed from crossing by the RA PU ( 0 , \pm 1 ) mode (Figure $3 \mathrm{~b}$, blue curve). Thus, the IR-140 PL band 
does not overlap significantly with the bonding mode but with the broad antibonding LSP mode ${ }^{14}$ centered around $910 \mathrm{~nm}$ that appears above $\theta>35^{\circ}$ (Figure 3a). As a result, the ESplasmon coupling region for $d=25 \mathrm{~nm}$ and $p=600 \mathrm{~nm} \mathrm{Au}$ bowties was defined above an in-plane wavevector of $3.2 \mu \mathrm{m}^{-1}$ (Figure $3 b$ ).

The mode volume (eq S1) supported by the 3D Au bowties at the bonding mode and antibonding LSP frequencies were calculated as $0.0004(\lambda / 2 n)^{3}$ and $0.0009(\lambda / 2 n)^{3}$, respectively (Supporting Information, Figure S3). We also calculated the mode volume of a $2 \mathrm{D}$ (planar) Au bowtie, which was shown to have an order-of-magnitude larger mode volume compared to a $3 \mathrm{D}$ bowtie at the bonding LSP wavelength (Supporting Information, Figure S4). Therefore, the 3D dimensionality of bowtie resonators is important for field-confinement effects. To test the field enhancement factors further under point dipole excitation (vs plane-wave excitation needed for explicit mode volume calculations), we performed FDTD simulations with a single dipole source to model the transition dipole of the gain molecule (Supporting Information, Figures S5-S7). Pointdipole excitation produced nearly the same field enhancement factor as that under plane-wave excitation at the LSP gap mode wavelength (Figure S5). As expected, when the point source was moved away from the gap region, the radiative and nonradiative coupling decay rates to the LSP mode were reduced substantially (Figure S6).

Since the mode volume of the antibonding mode is two times larger than that of the bonding mode, the lasing emission intensity for bowties with gaps of $25 \mathrm{~nm}$ is expected to be lower than those with gaps of $35 \mathrm{~nm}$. Indeed, the emission intensity at $882 \mathrm{~nm}$ was 10 times weaker (Figure $3 \mathrm{c}$ ). In addition, the fwhm of the laser emission was $6 \mathrm{~nm}$ (at $1.1 \mathrm{~mJ} \mathrm{~cm}^{-2}$ pump intensity), which is ca. 4 times wider than the $d=35 \mathrm{~nm}$ bowties. The wide lasing fwhm can be attributed to the broader width of the LSP resonance $\left(\Delta \omega_{\mathrm{SP}} \sim 100 \mathrm{~nm}\right)$. This line width broadening is qualitatively consistent with the SchawlowTownes relationship $\left(\Delta \omega_{\text {laser }} \propto\left(\Delta \omega_{\text {cavity }}\right)^{2} \equiv\left(\Delta \omega_{\mathrm{SP}}\right)^{2}\right){ }^{27}$

The 3D Au bowtie nanolaser arrays produced emission with $<2^{\circ}$ divergence at diffracted angle maxima $\theta_{\text {out }}=$ arcsin$\left(\left(m \lambda_{\text {laser }} / p\right)-\sin \theta_{\text {in }}\right)$, where $\lambda_{\text {laser }}$ is the outgoing laser wavelength, and $m$ specifies the order of interference. Figure $3 \mathrm{~d}$ demonstrates that, when the pump beam is incident with $\alpha=$ $-70^{\circ}$, for $p=600 \mathrm{~nm}$ bowtie arrays, two output beams are directed at the zeroth and first-diffraction angles, $70^{\circ}(m=0)$ and $-30^{\circ}(m=-1)$. If the bowties are independent and coherent oscillators, the directional beams can be attributed to in-phase interference between bowties that generates laser emission to principal maxima in the far-field. Collective effects from periodically spaced laser resonators have been observed in vertical cavity surface-emitting laser arrays. ${ }^{28,29}$ Besides LSPassisted lasing, band-edge emission was measured normal to the surface when the periodicity satisfied the condition for distributed feedback (DFB) lasing. Surface emission was independently observed at $912 \mathrm{~nm}$, close to the second-order grating condition, $m \lambda_{\text {laser }}=2 n_{\text {eff }} p$ where $m$ equals 2 (Figure 3d, inset). From the sample with $p=1200 \mathrm{~nm}$, however, surfacenormal DFB lasing cannot occur because that periodicity does not produce a DFB wavelength in the gain $\mathrm{PL}$ range. Furthermore, no lasing modes were found from patterned PU-IR140 samples without Au bowties (Supporting Information, Figure S2).

Femtosecond transient absorption (TA) measurements were carried out to determine the experimental Purcell factor for
LSP-assisted lasing. Figure 4a summarizes the Jablonski energy diagram of the ES-plasmon coupling process where IR-140
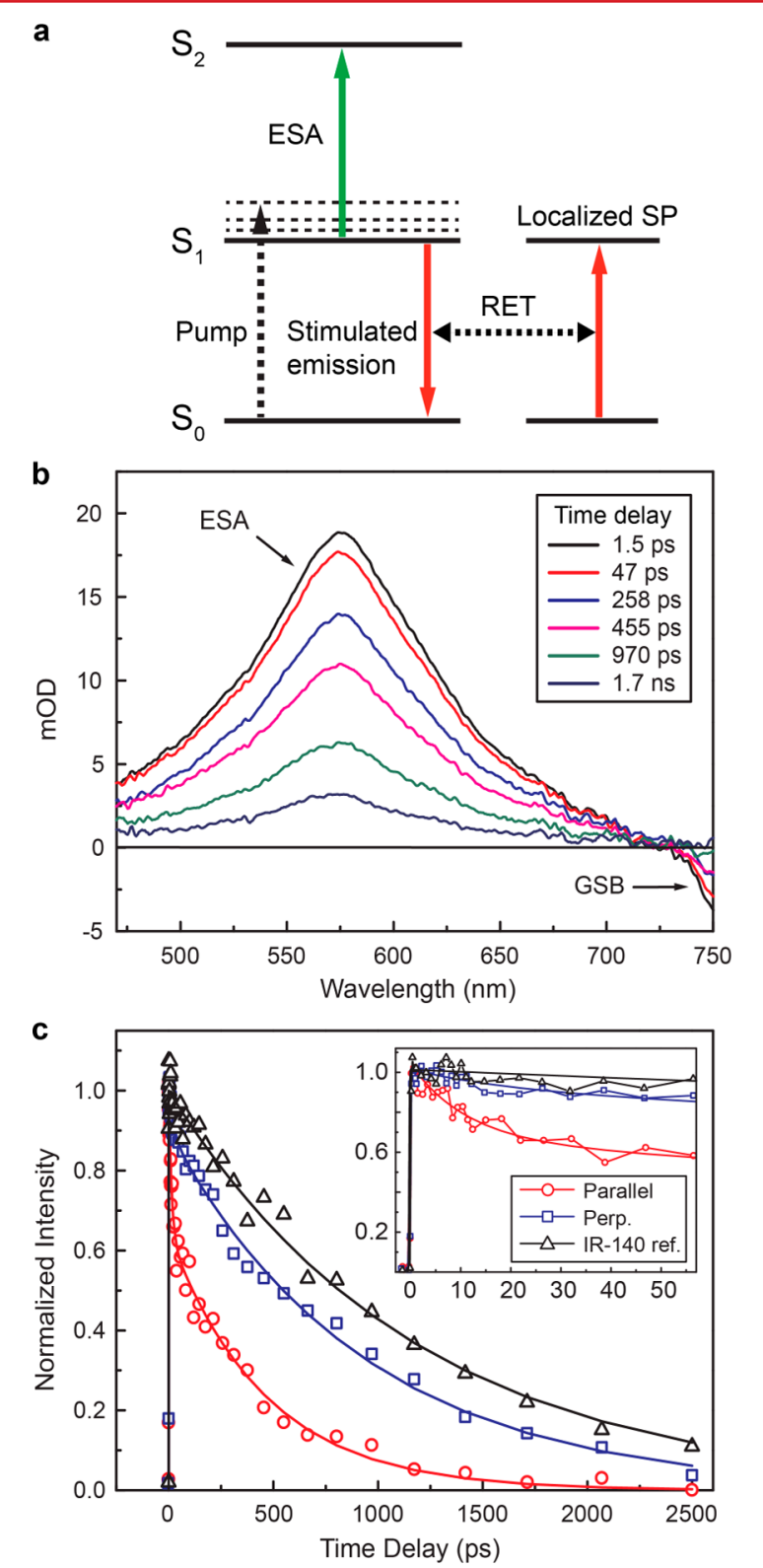

Figure 4. Pump-probe absorption measurements confirming the key process of SP-assisted lasing. (a) Jablonski energy diagram showing IR140 molecules photoexcited to the upper vibrational states of $S_{1}$. Resonance energy transfer then occurs between $S_{1}$ and the LSP mode. (b) Femtosecond TA spectra of the IR-140-only reference upon photoexcitation at $740 \mathrm{~nm}$. (c) Transient kinetics monitored at 580 $\mathrm{nm}$ under equivalent pumping conditions show a significant reduction in the $S_{1}$ lifetime of IR-140 because of the 3D Au bowties with $p=$ $1200 \mathrm{~nm}$. (inset) Initial fast ESA decays for pump polarization parallel, pump polarization perpendicular (perp), and a reference IR-140 sample in PU (ref).

molecules are excited by a pulsed laser to populate the first excited singlet state $\left(S_{1}\right)$. With the presence of $\mathrm{Au}$ bowties, energy is transferred to the LSP modes when the transition frequency of the dye coincides with that of the LSP resonance. Figure $4 \mathrm{~b}$ shows the TA spectra of the IR-140/PU-only reference as a function of pump-probe delay. Upon photoexcitation at $740 \mathrm{~nm}$, a broad ESA band centered at $580 \mathrm{~nm}$ 
and ground state bleach (GSB) feature at wavelengths $>730 \mathrm{~nm}$ were observed. With the IR-140 molecules in PU, a $\sim 1$ ns monoexponential decay of the ESA was measured. With the addition of the 3D Au bowties, TA spectra yielded identical spectral features, but new fast decay components were observed when pumped at parallel and perpendicular polarizations relative to the dimer axis.

The normalized transient kinetic traces monitored at $580 \mathrm{~nm}$ unambiguously demonstrate that the $\mathrm{Au}$ bowties induce ultrafast quenching of the excited state (Figure 4c). TA kinetics were fit to a sum of two exponentials, $I(t) \approx A_{1} \exp \left(-t / \tau_{1}\right)+$ $A_{2} \exp \left(-t / \tau_{2}\right)$. The fast decay time component when the pump beam was polarized parallel to the dimer axis was determined to be $\tau_{1}^{\text {par }}=13.4 \pm 4.5 \mathrm{ps}$, and $\tau_{1}^{\text {perp }}=23.9 \pm 15$ ps for perpendicular pump polarization. The slow decay components ( $\tau_{2}^{\text {par }}=\tau_{2}^{\text {perp }} \approx 1 \mathrm{~ns}$ ) were found to be equivalent to the intrinsic $S_{1}$ lifetime of IR-140 and represent the population of photoexcited dyes that did not participate in the RET process. ${ }^{20}$ The fast decay of the ES population indicates that the ESplasmon coupling introduced an ultrafast decay pathway. ${ }^{30}$

Although $\tau_{1}^{\text {par }}$ and $\tau_{1}^{\text {perp }}$ are identical within experimental error, the difference in relative amplitude ratios of the fast and slow components for the two pump polarizations is significant: $\left(A_{1} / A_{2}\right)_{\text {par }}=0.37$ and $\left(A_{1} / A_{2}\right)_{\text {perp }}=0.11$. The orientation of the dye molecules in the PU matrix is likely to be random; however, the polarization of the pump selectively excites molecules oriented such that their transition dipoles are parallel to the pump polarization. The large difference in the amplitude ratios of the ES-plasmon coupling confirms that the interactions between the localized plasmon fields and transition dipoles are stronger for dipoles parallel to the dimer axis than those that are perpendicular. This measured difference in coupling anisotropy is consistent with the large difference in lasing intensities for the two different pump polarizations (Figure 2b,c).

Importantly, the experimental Purcell enhancement factor determined by the ratio of the LSP coupling and radiative recombination rates $\left(F=k_{\mathrm{LSP}} / k_{\mathrm{rad}} \approx 300\right)$ agrees with the calculated Purcell factor from FDTD simulations $\left(F_{\mathrm{FDTD}} \approx 10^{3}\right.$, Supporting Information). The cavity loss per length $\alpha(\omega)$ is given by $\omega n / c Q^{31}$ where $n$ is the refractive index and $Q$ is the quality factor. For the $3 \mathrm{D}$ Au bowtie, the loss is estimated to be $4940 \mathrm{~cm}^{-1}$ using the experimentally measured quality factor $(Q$ $=35$, Supporting Information). Without the Au resonator, the gain $\left(N \sigma_{\mathrm{e}}=2640 \mathrm{~cm}^{-1}\right)$ cannot compensate the cavity loss. With the Au bowtie nanocavity, however, the emission crosssection $\left(\sigma_{\mathrm{e}}^{\mathrm{c}}=\sigma_{\mathrm{e}} F\right)$ is enhanced by the Purcell factor $(F=300)$. Therefore, the cavity-enhanced gain is greater than the loss $\left(N \sigma_{\mathrm{e}}^{\mathrm{c}} \gg \alpha(\omega)\right)$, which makes lasing action possible.

In summary, we have shown that subdiffraction nanoresonators based on metallic bowties, when coupled to a gain material, can generate coherent and directional light emission. In the absence of the bowties, the gain cannot overcome the losses of the system; however, because the emission crosssection is enhanced by the plasmonic cavity formed by the bowties, the gain is increased by the Purcell factor well above threshold, and lasing can occur at room temperature. Despite slight geometric inhomogeneities of individual nanolasers, we observed coherent light emission from the subdiffraction resonators; therefore, this bowtie design is highly defecttolerant, which is important for practical and scalable applications. Ultimately, the $3 \mathrm{D}$ resonator design can be interfaced with other gain materials, such as highly oriented molecules (e.g., J-aggregates) or single quantum dots, to improve the coupling efficiency for designing low-threshold nanolasers or new platforms for studying quantum plasmon effects.

\section{ASSOCIATED CONTENT}

\section{S Supporting Information}

Device fabrication, optical transmission, and transient absorption measurement; mode volume calculations; FDTD simulation with dipole source; spontaneous emission enhancement. This material is available free of charge via the Internet at http://pubs.acs.org.

\section{AUTHOR INFORMATION}

\section{Corresponding Author}

*E-mail: todom@northwestern.edu.

\section{Notes}

The authors declare no competing financial interest.

\section{ACKNOWLEDGMENTS}

This work was supported by the Initiative for Sustainability and Energy at Northwestern (ISEN) Award (J.Y.S., C.H.K., D.T.C.) and the NSF-MRSEC program at the Materials Research Science and Engineering Center at Northwestern University (DMR-1121262) (J.Y.S., W.Z., T.W.O.). M.D.H. acknowledges support by the Department of Defense through the National Defense Science and Engineering Graduate Fellowship (NDSEG) Program. TA measurement and data analysis were supported as part of the ANSER Center, an Energy Frontier Research Center funded by the U.S. Department of Energy, Office of Science, Office of Basic Energy Sciences (DESC0001059) (D.T.C. and M.R.W.).

\section{REFERENCES}

(1) Born, M.; Wolf, E. Principles of optics: electromagnetic theory of propagation, interference and diffraction of light, 7th expanded ed.; Cambridge University Press: New York, 1999; p xxxiii.

(2) Bergman, D. J.; Stockman, M. I. Surface plasmon amplification by stimulated emission of radiation: Quantum generation of coherent surface plasmons in nanosystems. Phys. Rev. Lett. 2003, 90, 027402.

(3) Gramotnev, D. K.; Bozhevolnyi, S. I. Plasmonics beyond the diffraction limit. Nat. Photonics 2010, 4, 83-91.

(4) Stockman, M. I. The spaser as a nanoscale quantum generator and ultrafast amplifier. J. Opt. 2010, 12, 024004.

(5) Berini, P.; De Leon, I. Surface plasmon-polariton amplifiers and lasers. Nat. Photonics 2012, 6, 16-24.

(6) Noginov, M. A.; Zhu, G.; Belgrave, A. M.; Bakker, R.; Shalaev, V. M.; Narimanov, E. E.; Stout, S.; Herz, E.; Suteewong, T.; Wiesner, U. Demonstration of a spaser-based nanolaser. Nature 2009, 460, 11101112.

(7) Hill, M. T.; Oei, Y. S.; Smalbrugge, B.; Zhu, Y.; De Vries, T.; Van Veldhoven, P. J.; Van Otten, F. W. M.; Eijkemans, T. J.; Turkiewicz, J. P.; De Waardt, H.; Geluk, E. J.; Kwon, S. H.; Lee, Y. H.; Notzel, R.; Smit, M. K. Lasing in metallic-coated nanocavities. Nat. Photonics 2007, 1, 589-594.

(8) Oulton, R. F.; Sorger, V. J.; Zentgraf, T.; Ma, R. M.; Gladden, C.; Dai, L.; Bartal, G.; Zhang, X. Plasmon lasers at deep subwavelength scale. Nature 2009, 461, 629-632.

(9) Ma, R. M.; Oulton, R. F.; Sorger, V. J.; Bartal, G.; Zhang, X. A. Room-temperature sub-diffraction-limited plasmon laser by total internal reflection. Nat. Mater. 2011, 10, 110-113.

(10) Kwon, S. H.; Kang, J. H.; Seassal, C.; Kim, S. K.; Regreny, P.; Lee, Y. H.; Lieber, C. M.; Park, H. G. Subwavelength Plasmonic Lasing from a Semiconductor Nanodisk with Silver Nanopan Cavity. Nano Lett. 2010, 10, 3679-3683. 
(11) Khajavikhan, M.; Simic, A.; Katz, M.; Lee, J. H.; Slutsky, B.; Mizrahi, A.; Lomakin, V.; Fainman, Y. Thresholdless nanoscale coaxial lasers. Nature 2012, 482, 204-207.

(12) Lu, Y. J.; Kim, J.; Chen, H. Y.; Wu, C. H.; Dabidian, N.; Sanders, C. E.; Wang, C. Y.; Lu, M. Y.; Li, B. H.; Qiu, X. G.; Chang, W. H.; Chen, L. J.; Shvets, G.; Shih, C. K.; Gwo, S. Plasmonic Nanolaser Using Epitaxially Grown Silver Film. Science 2012, 337, 450-453.

(13) Chang, S. W.; Ni, C. Y. A.; Chuang, S. L. Theory for bowtie plasmonic nanolasers. Opt. Express 2008, 16, 10580-10595.

(14) Suh, J. Y.; Huntington, M. D.; Kim, C. H.; Zhou, W.; Wasielewski, M. R.; Odom, T. W. Extraordinary Nonlinear Absorption in 3D Bowtie Nanoantennas. Nano Lett. 2012, 12, 269-74.

(15) Yu, N. F.; Cubukcu, E.; Diehl, L.; Bour, D.; Corzine, S.; Zhu, J. T.; Hofler, G.; Crozier, K. B.; Capasso, F. Bowtie plasmonic quantum cascade laser antenna. Opt. Express 2007, 15, 13272-13281.

(16) Sundaramurthy, A.; Crozier, K. B.; Kino, G. S.; Fromm, D. P.; Schuck, P. J.; Moerner, W. E. Field enhancement and gap-dependent resonance in a system of two opposing tip-to-tip $\mathrm{Au}$ nanotriangles. Phys. Rev. B 2005, 72, 165409.

(17) Zhang, S.; Park, Y. S.; Liu, Y. M.; Zentgraf, T.; Zhang, X. Farfield measurement of ultra-small plasmonic mode volume. Opt. Express 2010, 18, 6048-6055.

(18) Kuttge, M.; de Abajo, F. J. G.; Polman, A. Ultrasmall Mode Volume Plasmonic Nanodisk Resonators. Nano Lett. 2010, 10, 15371541.

(19) Chang, S. W.; Chuang, S. L. Fundamental Formulation for Plasmonic Nanolasers. IEEE J. Quantum Electron. 2009, 45, 10041023.

(20) Sperber, P.; Spangler, W.; Meier, B.; Penzkofer, A. Experimental and Theoretical Investigation of Tunable Picosecond Pulse Generation in Longitudinally Pumped Dye-Laser Generators and Amplifiers. Opt. Quant. Electron. 1988, 20, 395-431.

(21) Djiango, M.; Kobayashi, T.; Blau, W. J. Cavity-enhanced stimulated emission cross section in polymer microlasers. Appl. Phys. Lett. 2008, 93, 143306.

(22) Huang, J. S.; Kern, J.; Geisler, P.; Weinmann, P.; Kamp, M.; Forchel, A.; Biagioni, P.; Hecht, B. Mode Imaging and Selection in Strongly Coupled Nanoantennas. Nano Lett. 2010, 10, 2105-2110.

(23) Purcell, E. M. Spontaneous Emission Probabilities at Radio Frequencies. Phys. Rev. 1946, 69, 681-681.

(24) Kravets, V. G.; Schedin, F.; Grigorenko, A. N. Extremely narrow plasmon resonances based on diffraction coupling of localized plasmons in arrays of metallic nanoparticles. Phys. Rev. Lett. 2008, 101, 087403.

(25) Vecchi, G.; Giannini, V.; Rivas, J. G. Surface modes in plasmonic crystals induced by diffractive coupling of nanoantennas. Phys. Rev. $B$ 2009, 80, 201401.

(26) De Leon, I.; Berini, P. Amplification of long-range surface plasmons by a dipolar gain medium. Nat. Photonics 2010, 4, 382-387.

(27) Schawlow, A. L.; Townes, C. H. Infrared and Optical Masers. Phys. Rev. 1958, 112, 1940-1949.

(28) Lehman, A. C.; Raftery, J. J.; Carney, P. S.; Choquette, K. D. Coherence of photonic crystal vertical-cavity surface-emitting laser arrays. IEEE J. Quantum Electron. 2007, 43, 25-30.

(29) Warren, M. E.; Gourley, P. L.; Hadley, G. R.; Vawter, G. A.; Brennan, T. M.; Hammons, B. E.; Lear, K. L. On-Axis Far-Field Emission from 2-Dimensional Phase-Locked Vertical Cavity SurfaceEmitting Laser Arrays with an Integrated Phase-Corrector. Appl. Phys. Lett. 1992, 61, 1484-1486.

(30) Barnes, W. L. Fluorescence near interfaces: the role of photonic mode density. J. Mod. Opt. 1998, 45, 661-699.

(31) Yariv, A. Optical electronics in modern communications, 5th ed.; Oxford University Press: New York, 1997; p xviii. 